\title{
A Search Procedure and Lower Bound for Odd Perfect Numbers
}

\author{
By Bryant Tuckerman
}

\begin{abstract}
An infinite tree-generating " $q$-algorithm" is defined, which if executed would enumerate all odd perfect numbers (opn's). A truncated execution shows that any opn has either some component $p^{a}>10^{18}$, with $a$ even, or no divisor $<7$; hence any opn must be $>10^{36}$.
\end{abstract}

1. Introduction. It is unknown whether any odd perfect numbers (opn's) exist. For a history, see McCarthy [2]. Kanold [3] gave a lower bound of $10^{20}$ for possible opn's. Muskat [5] showed that every opn must have a divisor $p^{a}>10^{12}$.

The present paper is a condensed and clarified version of [6], which was announced in [7], and submitted to this journal in 1968. The completion of the requested revision has been delayed until now.

The chief results are:

(I) A " $q$-algorithm" is given, which defines a countably infinite tree, on which, if enumerated, every opn (if any) would be recognized at some node.

(II) A finite truncation of the tree was computed, which shows that (1) every opn must satisfy the known restrictions defined at some one of the truncation-nodes implied by this tree; (2) every opn must have either (a) some component $p^{a}>u=$ $10^{18}$, with $a$ even, or (b) no prime divisor $<7$; (3) hence any opn must be $>u^{2}=10^{36}$.

This " $10^{36}$-tree", occupying 9 pages, has been deposited in the UMT file [8] (and occurs in an earlier arrangement in [6]). To convey the spirit of that tree and of the algorithm, an analogous " $10^{16}$-tree" (based on $u=10^{8}$ ), occupying 2 pages, is included in this paper. Statements about the $10^{36}$-tree will typically hold equally well for the $10^{16}$-tree.

The bound of $10^{36}$ has been superseded in a recent paper by Hagis [9]. Nevertheless, there are enough different approaches in the two papers to warrant the present publication. For omitted proofs and details, see [6].

2. Notation. Let $N, P, \Omega$ be the sets of all positive integers $(n, m, h, d$, etc.), primes $(p$ or $q)$, and opn's, respectively. Let $a(p, n) \geqq 0$ be the multiplicity of $p$ in $n$, and let $h(p, n)=a(p, n)+1 \geqq 1$. With Sylvester, we call $p^{a(p, n)}>1$ a component of $n$. Let $A(n)=\{p: p \mid n\}$. For $n>1$, define $p_{1}(n)=\min \{p \in A(n)\}$; the other $p \in A(n)$ may be numbered $p_{2}(n), \cdots, p_{r(n)}(n)$ in any convenient order. We may then represent $n$ in any of the forms

$$
n=\prod_{i=1}^{r(n)} p_{i}(n)^{a_{i}(n)}=\prod_{i=1}^{r(n)} p_{i}(n)^{h_{i}(n)-1}=\prod_{p} p^{a(p, n)}=\prod_{p} p^{h(p, n)-1}
$$

where the range of $p$ in the last two products is indifferently $P$ or $A(n)$.

Received April 29, 1968, revised June 19, 1972.

AMS (MOS) subject classifications (1970). Primary 10A40, 10A25, 10A99, $10-04$.

Key words and phrases. Odd perfect numbers, perfect numbers.

Copyright (c) 1973, American Mathematical Society 
Given $p^{a}$, write $h=a+1$. Then $\sigma\left(p^{a}\right)=\sigma\left(p^{h-1}\right)=\left(p^{h}-1\right) /(p-1)=\prod_{d \in D} F_{d}(p)$ where $F_{d}(\cdot)$ is the $d$ th cyclotomic polynomial (see Nagell [1]), and $D=\{d: d \mid h \wedge$ $d \neq 1\}$. If $h_{1} \mid h_{2}$, then $\sigma\left(p^{h_{1}-1}\right) \mid \sigma\left(p^{h_{2}-1}\right)$ (proof in [6, p. 23]). Thus in using $\sigma(\cdot)$ and in studying opn's, it is more effective to deal with $h$ and its prime divisors rather than with $a$. It will prove convenient to write its prime factorization as $h=q_{1} q_{2} \cdots q_{s}$, where $q_{1} \leqq q_{2} \leqq \cdots \leqq q_{s}$. By the $j$ th prime divisor of $h$ we will mean $q_{j}$. (Thus if $h=45=3 \cdot 3 \cdot 5$, then the 1st, 2nd, 3rd prime divisors of $h$ are 3, 3, 5.) For $h=$ $h(p, n)$, we define $s(p, n)$ and $q_{i}(p, n)$ like $s$ and $q_{i}$.

We say $n_{1} h$-divides $n_{2},\left.n_{1}\right|_{h} n_{2}$, if for all $p, h\left(p, n_{1}\right) \mid h\left(p, n_{2}\right) ; n_{1} q$-divides $n_{2},\left.n_{1}\right|_{e} n_{2}$, if for all $p, s\left(p, n_{1}\right) \leqq s\left(p, n_{2}\right)$ and $q_{i}\left(p, n_{1}\right)=q_{i}\left(p, n_{2}\right)$ for $1 \leqq j \leqq s\left(p, n_{1}\right)$. Clearly, $\left.n_{1}\right|_{a} n_{2}$ implies $\left.n_{1}\right|_{h} n_{2}$.

LEMMA. If $\left.n_{1}\right|_{a} n_{2}$, or if $\left.n_{1}\right|_{h} n_{2}$, then $n_{1} \mid n_{2}$ and $\sigma\left(n_{1}\right) \mid \sigma\left(n_{2}\right)$.

This result accounts for the usefulness of the concepts $\left.\right|_{h}$ and $\left.\right|_{a}$. The proof is easy.

In the context of opn's, we define a prime power $p^{a}>1$ to be ordinary if $a \equiv$ $0 \bmod 2$ (i.e. $h$ is odd); exceptional if $p \equiv 1 \bmod 4$, and $a \equiv 1 \bmod 4$ (i.e., $h$ is singly even); inadmissible otherwise. For $k=0,1,2, \cdots$, define $E_{k}=\{n$ : exactly $k$ of the components of $n$ are exceptional, and the rest are ordinary\}. Euler proved that $\Omega \subset E_{1}$.

3. Trees. Let $C$ be a set of choices $c ; \lambda$ a fixed symbol; $C^{*}=\left\{\nu: \nu=\lambda c_{1} c_{2} \cdots c_{l}\right.$ where $\left.c_{i} \in C\right\}\left(l=l_{\nu} \geqq 0\right.$ is called the level of $\left.\nu\right)$; and $C(\cdot)$ a function $C(\cdot): C^{*} \rightarrow 2^{C}$ (we denote the image $C(\nu)$ by $C_{\nu}$ ). Then a unique tree $T=T_{C(\cdot)}$, rooted at $\lambda$, is defined as the smallest set $T$ of nodes $\nu$ such that $\lambda \in T$, and such that if $\nu=\lambda c_{1} c_{2} \cdots c_{l} \in T$, then $\nu c=\lambda c_{1} c_{2} \cdots c_{l} c_{l+1} \in T$ for all $c=c_{l+1} \in C_{\nu}$.

4. Opn-Trees. We will define such a $T$, called an opn-tree, with the following properties.

At each $\nu \in T$, of level $l$, there will be defined a sequence $R_{\nu}=\left(\rho_{1}, \cdots, \rho_{l}\right)$ of $l$ restrictions (truth-valued functions) $\rho_{k}(n)$ on the variable $n$. Let $N_{\nu}=\left\{n: \rho_{k}(n)\right.$ is true for all $\left.\rho_{k} \in R_{\nu}\right\}$; let $\Omega_{\nu}=N_{\nu} \cap \Omega$. Clearly $R_{\lambda}=\varnothing, N_{\lambda}=N, \Omega_{\lambda}=\Omega$. At some $\nu$, it might become known that $\Omega_{\nu}=\{n\}$ where $n$ is known to be an opn. We will call this $\nu$ an opn-node. At some other $\nu$ it may become known that $\Omega_{v}=\varnothing$. In either case, $\nu$ is a terminal node, and we do not branch from it. Otherwise, we will select, and branch on, some function $f_{v}(n)$, known to be defined for all $n \in \Omega_{\nu}$, with "adequate" range $C_{\nu}$, i.e., such that $f_{\nu}\left(\Omega_{\nu}\right) \subseteq C_{\nu} \subseteq C$. Thus, if we define the sons of $\nu$ to be all $\nu c$ for $c \in C_{\nu}$, then at each $\nu c$ we define $R_{v c}=\left(\rho_{1}, \cdots, \rho_{l}, \rho_{l+1}\right)$ where $\rho_{l+1}(n)$ is ' $f_{\nu}(n)=c$ '. Clearly, $\left\{\Omega_{\nu c}: c \in C_{\nu}\right\}$ is a disjoint partitioning of $\Omega_{\nu}$. Hence, every $m \in \Omega$ (if any) lies on a unique path from $\lambda$ down $T$.

At $\nu=\lambda$, we branch on $p_{\min }(n)$, with $C_{\nu}=P-\{2\}$. At every other $\nu \in T$ of level $l>0$, we will branch (if at all) on $q_{i l}\left(\hat{p}_{l}, n\right)$, i.e., on the $j_{l}$ th prime divisor of $h\left(\hat{p}_{l}, n\right)$, for suitable $\hat{p}_{l}, j_{l}$ (both depending on $\nu$ ) and $C_{\nu}$. As a result, at every $\nu=$ $\lambda c_{1} c_{2} \cdots c_{l} \in T$ of level $l>0$ we will have $R_{v}=\left(\rho_{1}, \rho_{2}, \cdots, \rho_{l}\right)$ where

$$
\begin{aligned}
& \rho_{1} \quad \text { is } \quad p_{\min }(n)=c_{1} \text { ', } \\
& \rho_{k} \quad \text { is ' } q_{i_{k-1}}\left(\hat{p}_{k-1}, n\right)=c_{k} \text { ' for } k=2,3, \cdots, l \text {. }
\end{aligned}
$$

Of course, $\hat{p}_{k-1}, j_{k-1}$, and a $C_{\nu_{k-1}}$ were defined at $\nu_{k-1}=\lambda c_{1} c_{2} \cdots c_{k-1}$, and $c_{k} \in C_{\nu_{k-1}}$. 
The sequence $\hat{p}_{1}, \hat{p}_{2}, \cdots, \hat{p}_{l-1}$ may contain repetitions. Let its distinct elements, in order of first appearance, be $p_{1}, p_{2}, \cdots, p_{r}$, and let $A_{v}=\left\{p_{i}\right\}$. The later conditions on branching will ensure that $p_{1}=c_{1}$ and that, for each $p_{i}$, the subsequence of the $\rho_{k}(2 \leqq k \leqq l)$, for which $\hat{p}_{k-1}=p_{i}$, will have consecutive values $1,2, \cdots, s_{i}(i \geqq 1)$ of $j_{k-1}$, and nondecreasing prime values of $c_{k}$. Consequently, we can replace $R_{v}$ by the equivalent

$$
\begin{aligned}
& p_{\min }(n)=p_{1}, \\
& q_{i}\left(p_{i}, n\right)=q_{i j} \text { for } i=1,2, \cdots, r ; j=1,2, \cdots, s_{i},
\end{aligned}
$$

where $p_{1}=c_{1}, r$ and the $s_{i}$ are known $\left(\sum s_{i}=l-1\right)$, and the $q_{i j}$ are a known permutation of the $c_{k}(k>1)$; or equivalently

$$
\begin{aligned}
& p_{\min }(n)=p_{1}, \\
& \left.m\right|_{a} n,
\end{aligned}
$$

where $m=m_{\nu}=\prod_{i=1}^{r} p_{i}^{h_{i}-1}$ and $h_{i}=\prod_{i=1}^{s_{i}} q_{i}$.

All of $l, \hat{p}_{k-1}, j_{k-1}, c_{k}, r, p_{i}, s_{i}, q_{i j}, h_{i}, m$ are functions of $\nu$. Since we generally consider a typical $\nu$ and its sons $\nu c$ or father $\nu^{-}$, we omit this dependence on $\nu$ from the notation, except that for $l=l_{\nu}$ and $m=m_{\nu}$ it is optional. Such sets as $N_{\nu}, \Omega_{\nu}, A_{\nu}$ will always bear the subscript.

5. The Computation of $\sigma(m)$. For $l=1, \sigma(m)=m=1$. For $l>1$, we are assisted in calculating $\sigma(m)$ by the assumed previous factorization of $\sigma\left(m^{-}\right)$, where $m^{-}=m_{\nu}$. For that $i$ such that $p_{i}=\hat{p}_{l-1}$, write $p$ for $p_{i}, s$ for $s_{i}, q$ for $q_{i s}, h$ for $h_{i} / q$. Then $p$ appears in $m^{-}$as $p^{h-1}$ (possibly as $p^{0}=1$ ) and in $m$ as $p^{h q-1}$. Thus, $\sigma(m) / \sigma\left(m^{-}\right.$) $=\sigma\left(p^{h_{q-1}}\right) / \sigma\left(p^{h-1}\right)=\left(p^{h a}-1\right) /\left(p^{h}-1\right)=\prod_{d \in D} F_{d}(p)$, where $D=\{d: d \mid h q \wedge$ $d \nmid h\}$. The factorization of $\sigma(m)$ may therefore be found from that of $\sigma\left(m^{-}\right)$and of each $F_{d}(p)$. In the important special case $s=1, h=1, p \nmid m^{-}$we have simply $\sigma(m) / \sigma\left(m^{-}\right)=\sigma\left(p^{q-1}\right)=F_{q}(p)$. In the $10^{36}$-tree, only this case arose.

If we define $b(p, m)=a(p, \sigma(m))$, then $\sigma(m)=\prod_{p} p^{b(p, m)}$. Define $B_{\nu}=\{p \neq$ $2: b(p, m)>0\}$. We will ensure, inductively, that $m \in E_{0} \cup E_{1}$. (For $l \leqq 1, m=$ $1 \in E_{0}$.) If $m \in E_{0}$, then no $q_{i j}=2$, and $b(2, m)=0$. If $m \in E_{1}$, then just one $q_{i j}=2$, for some $i$, and $j=1 ; p_{i}$ is the exceptional prime, and $b(2, m)=1$.

6. More Details on Branching. At each node $\nu \neq \lambda$ we define a set $P_{\nu}$ of $p$ which are admissible to be $\hat{p}=\hat{p}_{l}$. If $l=1$, let $P_{\nu}=\left\{p_{1}\right\}$, where $p_{1}=c_{1}$. If $l>1$, let $P_{\nu}=\{p \neq 2: b(p, m)>a(p, m)\}$, i.e., the set of $p \neq 2$ which are "over-generated" by $\sigma(\cdot)$. The proof of our next result may be found on p. 29 of [6].

THEOREM. If $l_{\nu} \geqq 1$, and $P_{\nu}=\varnothing$, then $m=m_{\nu}$ is an opn, and $\Omega_{\nu}=\{m\}$. Thus $\nu$ is an opn-node.

Otherwise, consider any $p \in P_{\nu} \neq \varnothing$. For every $n \in \Omega_{\nu}, a(p, n)=b(p, n) \geqq$ $b(p, m)$. If $l>1, b(p, m)>a(p, m)$. Hence, $a(p, n)>a(p, m)$. The latter also holds for $l=1$. Thus, $h(p, n)>h(p, m)$; and since $\left.m\right|_{a} n, s(p, n)>s(p, m)$. Thus, $q_{s(p, m)+1}(p, n)$ is defined for all $n \in \Omega_{\nu}$, so that it is admissible to branch on it. There are two cases.

If $p \notin A_{\nu}$ (in particular, if $l=1$ ), then $s(p, m)=0$. It is thus admissible to branch 
on $q_{1}(p, n)$, with $C_{\nu}=P$ if $p$ is admissible as the exceptional prime, i.e., if $p \equiv 1 \bmod 4$ and $m \in E_{0}$. Otherwise, $C_{\nu}=P-\{2\}$.

If $p \in A_{v}$, say $p=p_{i}$, then $s\left(p_{i}, m\right)=s_{i}>0$. It is then admissible to branch on $q_{s i+1}\left(p_{i}, n\right)$, with $C_{v}=\left\{q \in P: q \neq 2 \wedge q \geqq q_{i s_{i}}\right\}$.

The use of any of the above $p$ as $\hat{p}_{l}$ preserves, at all $\nu c$, the properties assumed at $\nu$. In particular, if $c=2$, then $\nu \in E_{0}$ and $\nu 2 \in E_{1}$; otherwise, $\nu$ and $\nu c$ both belong to $E_{0}$ or both belong to $E_{1}$. Thus the property $m \in E_{0} \cup E_{1}$ at $\nu$ is preserved at all $\nu c$.

Any well-defined global selection rule(s) (i.e., for all $\nu \in T$ ) for $\hat{p} \in P_{\nu} \neq \varnothing$ will define a particular tree. A set of such rules is given on p. 40 of [6]. In the $10^{36}$-tree, these rules always reduced (for $l>1)$ to $\hat{p}=\max \left\{p \in\left(B_{v}-B_{v}-\right)\right\}$; and always $\hat{p} \notin A_{v}$. Hence, the branching was always on $q_{1}(\hat{p}, n)$, so that always $s_{i}=1, p_{i}=\hat{p}_{i}$, $h_{i}=q_{i 1}(i=1,2, \cdots, r), r=l-1$; but the more general case may be needed for the infinite tree.

Thus an opn-tree is defined. Its previously assumed properties hold, by induction on $l$. It is enumerable, in fact by various admissible sequences, in which the father $\nu$ of any $\nu c$ is processed before $\nu c$.

If $n$ is any opn, it lies on a unique path from $\lambda$, and will be recognized at the node $\nu$ on this path which has level $l=1+\sum_{i=1}^{r(n)} s\left(p_{i}, n\right)$.

7. Contradiction-Nodes. This tree can be pruned, during construction, by taking into consideration two conditions which can give contradictions to $n \in \Omega_{\nu}$, thus showing that $\Omega_{\nu}=\varnothing$, so that we can make $\nu$ a terminal node. These are discussed in the next two paragraphs.

For every $p \in B_{v}, p|\sigma(m)| \sigma(n)=2 n$, for all $n \in \Omega_{\nu}$. Hence if $\exists p \in B_{\nu}$ such that $p<p_{1}$, this contradicts $p_{1}=p_{\mathrm{min}}(n)$, and we have a least-element-contradictionnode at $\nu$.

For every $n \in \Omega_{\nu}$, (1) $\left.m\right|_{a} n$, (2) $\sigma(m) \mid 2 n$, (3) $n \in E_{1}$. Let $N_{\nu}^{\prime}=\{n:$ (1) $\wedge$ (2) $\wedge$ $(n \in E) \wedge\left(n \in E_{1}\right.$ if $\left.\left.m \in E_{1}\right)\right\}$, where $E=\bigcup_{k=0}^{\infty} E_{k}$. Let $m^{\prime}=\min \left\{n \in N_{\nu}^{\prime}\right\}=$ $\operatorname{gcd}\left\{n \in N_{\nu}^{\prime}\right\} ; m^{\prime}$ is readily determined by its $\left\{a\left(p, m^{\prime}\right)\right\}$ in terms of $\{a(p, m)\}$ and $\{b(p, m)\}$. (Cf. [6, pp. 37-39]; and note, for example, that $2=a(p, m)<b(p, m)(\leqq 8)$ implies $a\left(p, m^{\prime}\right)=3^{2}-1=8$, a strong contribution to $m^{\prime}$, and to $\sigma\left(m^{\prime}\right) / m^{\prime}$, especially for $p=3$.) Now $m^{\prime} \mid n$ for all $n \in \Omega_{\nu}$. If we compute $\sigma\left(m^{\prime}\right) / 2 m^{\prime}$, and if this is $>1$, then $m^{\prime}$ is abundant, and so are its multiples $n$. Hence, $\Omega_{\nu}=\varnothing$, and $\nu$ is an abundancecontradiction-node. (The $10^{36}$-tree was first computed without the use of abundancecontradictions and had about twice as many nodes, and about twice the depth, as with their use.)

Although not used as such in [6], the above $m^{\prime}$ is a lower bound for all $n \in \Omega_{v}$; and a "better" bound is $m^{\prime \prime}=\min \left\{n:(1) \wedge(2) \wedge\left(n \in E_{1}\right)\right\}$, which is $>m^{\prime}$ if $m^{\prime} \notin E_{1}$.

8. Truncated Execution. For computation, the opn-tree must be truncated to a finite tree. For simplicity, and because the chief computational effort was in factoring large $F_{a}(p)$, it was decided to truncate primarily by omitting all $v q$ (truncation nodes) for which $p^{h-1}>u$ or $>2^{1 / 2} u$, according as $h q$ is odd, or even and $>2$, for a chosen $u$. Any opn at such a node is $>u^{2}$. To obtain the same bound at level 1 , we used results of Norton [4] and derived (pp. 35-36 of [6]) these lower bounds $w\left(p_{\min }(n)\right): w(3)=10^{3} ; w(5)=10^{13} ; w(7)=10^{41} ; w(11)=10^{90} ; w(13)=10^{154}$; etc. We let $\lambda p$ be truncation nodes as soon as $w(p)>u^{2}$. Using $u=10^{18}$ and then $u=10^{8}$, with $\max \left(p_{\min }\right)=5$ in both cases, yielded the $10^{36}$ - and $10^{16}$-trees. 
ODD-PERFECT-NUMBER SEARCH TREE TO $10 * * 16$

(REPRESENTATIVE OF SEARCH TREE TO 10**36)

NDDE $L E F$

$\begin{array}{ll}3 & 0 \text { - ALL ODD PERFECT NUMBERS } \\ 33 & 1 \text { - LEAST ELEMENT 3, HENCE N>10**3 }\end{array}$

$332 \quad 3 * F(3,3)=13$

$3323 \quad 4 \quad F(3,7)=3.19$

$33233 \quad 5 \quad F(3,19)=3.127$

3323336 F( 3,127$)=3.5419$

3323333

3323333*

33235

332353

33237

3325

33253

332533

3325333

33253333

33253333*

3327

33273

333

3332

33323

333233

3332333

33325

33325*

3333

33332

333323

333323*

333325

3333253

33332533

33332533*

333327

3333273

33333

333332

333332 *

333333

3333332

33335

333352

3333523

33335233

3335

$F(3,5419)=3.31 .313 .1009$

$3 * * 8 .(7.19 .31) * * 2.13 * * 1$

$+.024283$

$F(5,19)=151.911$

$F(3,911)=830833$

$F(7,19)=701.70841$

$F(5,7)=2801$

$F(3,2801)=37.43 .4933$

$F(3,4933)=3.127 .193 .331$

$F(3,331)=3.7 .5233$

$F(3,5233)=3.7 .31 .42073$

$3 * * 8 \cdot 7 * * 4 .(31.37) * * 2.13 * * 1$

171

$F(7,7)=29.4733$

$F(3,4733)=22406023$

- $F(3,13)=3.61$

* $F(2,61)=2.31$

$F(3,31)=3.331$

$F(3,331)=3.7 .5233$

$F(3.5233)=3.7 .31 .42073$

$F(5,31)=5.11 .17351$

$(3 \cdot 5 \cdot 11 \cdot 13) * * 2$

- $F(3.61)=3.13 .97$

* $F(2,97)=2.7 * * 2$

$F(3,7)=3.19$

$3 * * 8 .(7.13 \cdot 19.61) * * 2$

$F(5,7)=2801$

$F(3,2801)=37.43 .4933$

$F(3,4933)=3.127 .193 .331$

$3 * * 8.7 * 4 .(13.37 .43 .61) * * 2$

$1331^{\circ}$

$F(7,7)=29.4733$

$F(3,4733)=22406023$

- $F(3,97)=3.3169$

6 * F( 2.3169$)=2.5 .317$

$3 * * 8 \cdot(5.13) * * 2$

$6-F(3.3169)=3.3348577$

$7 * F(2,3348577)=2.1674289$

5 - $F(5,97)=11.31 .262321$

$6 * F(2,262321)=2.31 .4231$

$F(3,4231)=3.601 .9931$

$F(3,9931)=3.211 .155821$

$-F(5.61)=5.131 .21491$

The number of nodes in the $10^{36}$-tree could have been reduced somewhat, and the amount of computation reduced somewhat more, by using the $m^{\prime \prime}$ mentioned earlier. This was not done, partly for simplicity, partly to obtain the statement $p^{a}>u=10^{18}$ in the Introduction, and partly not to limit the search for possible opn's unnecessarily soon.

9. Description of the Listings. The nodes are in lexicographic order. The field "NODE" shows $c_{1} c_{2} \cdots c_{l}$ (omitting $\lambda$ ), with the primes $11,13,17, \cdots$ abbreviated as $A, B, C, \cdots$. " $L$ " shows $l$. " $E$ " contains (-, *, blank) according as 


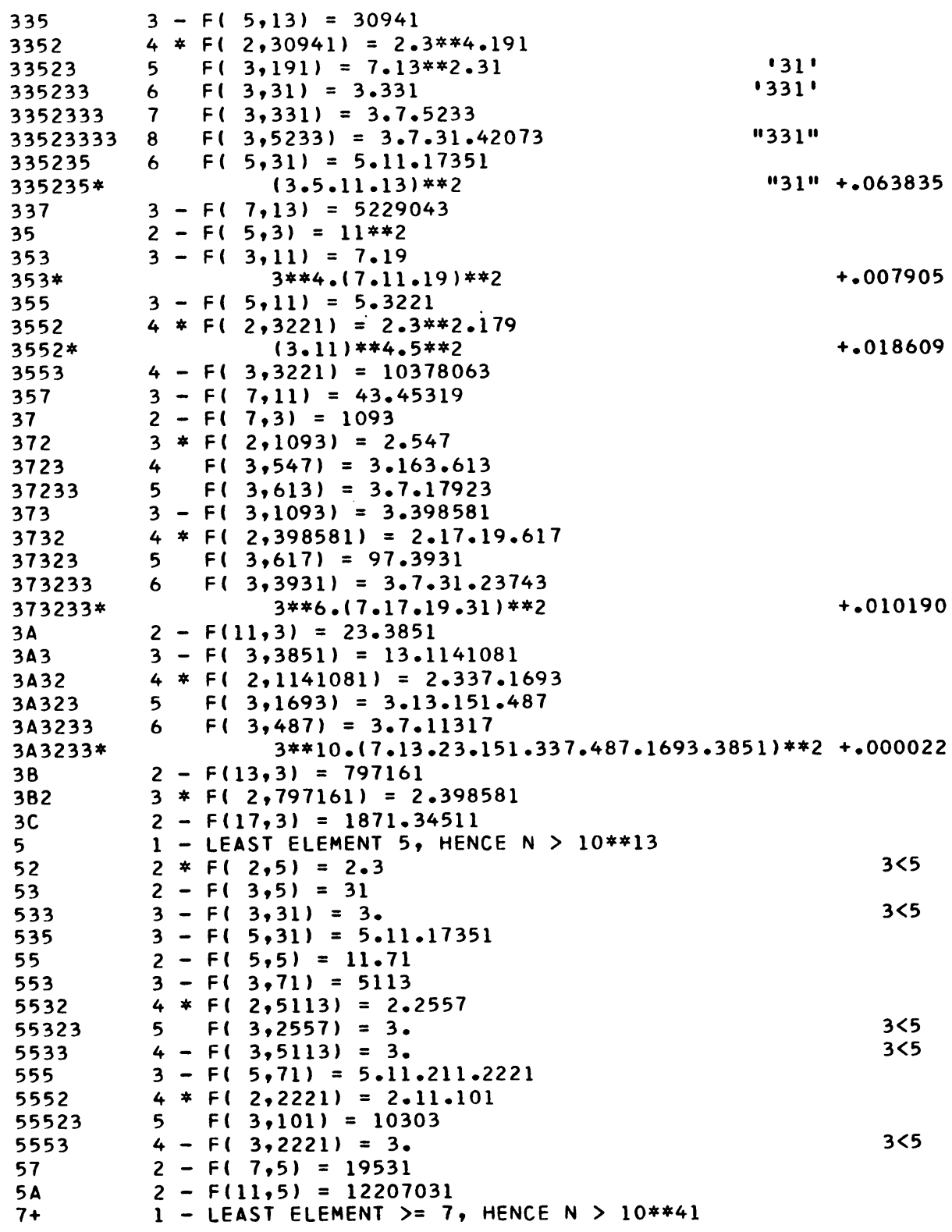

the exceptional prime has (not yet, just, already) been chosen. " $F$ " shows $F(q, p)=$ $F_{q}(p)$ and its factorization, performed on an IBM 7094. If $\nu$ has an abundancecontradiction, the succeeding line $\nu^{*}$ shows an abundant $\bar{m} \mid m^{\prime}$, and the value of $\ln (\sigma(\bar{m}) / 2 \bar{m})>0$. For a fuller explanation, see pp. 37-39 and 45 of [6]. I am indebted to a referee for four minor corrections to the hand-computed $\bar{m}$. Least-elementcontradictions are indicated by " $3<5$ ". Any branch-nodes not shown, of other than terminal nodes, are truncation nodes. The presentation of the $10^{36}$-tree [8] completes the demonstration of the assertions in the Introduction.

For $\hat{p}=7,31,331$ there are $2,2,3$ nodes $\nu^{(\hat{\nu}, i)}$ having the same branching func- 
tion, range, and $p_{\min }(n)$ for each $i$. For example, at both $\nu^{(7,1)}=\lambda 332$ and $\nu^{(7,2)}=$ $\lambda 33332$, the branching is on $q_{1}(7, n)$. For any such $\hat{p}$, the subtrees rooted at each $\nu^{(\hat{p}, i)}$ will have identical branchings and factorizations (at least initially), aside from abundance-contradictions which merely cause different prunings of these subtrees.

The major benefit of identifying these subtrees is to eliminate duplicate execution of some factorizations. A lesser potential benefit could be to shorten the listings. In [6], some shortening was obtained by "overlaying" all the subtrees for each such $\hat{p}$ onto one of them, on which the separate cases were carried along. In the present listings, for greater clarity these subtrees have not been overlaid. However, each has been demarcated by ' $\hat{p}$ ' at its root, and " $\hat{p}$ " at its lexicographically last node.

Some space, but not much computation, could be saved by subsuming the cases for such a $\hat{p}$ into one appropriate new case (represented on a separate tree) which includes appropriate common restrictions. For example, the cases for $\hat{p}=7$ could be subsumed into a case with the restrictions $p_{\min }(n)=3, m=\left.3^{2} \cdot\left(13^{1}\right.$ or $\left.97^{1}\right)\right|_{a} n$ (hence $\left.m \in E_{1}\right), 2^{1} \cdot 3^{1} \cdot 7^{1} \cdot 13^{1} \mid \sigma(n)$; branch on $q_{1}(7, n)$. Analogs of $m_{v}^{\prime}$ and $m_{v}^{\prime \prime}$ could be defined; for example, let $m^{\prime}=m^{\prime \prime}=3^{2} \cdot 7^{2} \cdot 13^{1}$ at the root.

10. A Comparison. The above $q$-algorithm is a modification of a simpler " $a$-algorithm" [6] which uses branchings on $a(\hat{p}, n)$. The "complication" is more than compensated for by the fewer branches and factorizations. For example, with $u=10^{18}$, the branches at $\lambda 3$, with $\hat{p}=3$, are reduced from 18 cases of $\hat{p}^{a}<u$ to 11 cases of $\hat{p}^{a_{1}-1}<u$; at $\lambda 33$, with $\hat{p}=13$, from 12 cases to 7 ; at $\lambda 5$, with $\hat{p}=5$, from 19 cases to 9. Each omitted case, i.e., one for which $h=a+1$ is composite, is subsumed in the case of the least $q \mid h$, with retention of the useful common factorization of $F_{q}(\hat{p})$, and omission of the not-yet-needed further branching and computation.

I am indebted to a referee for significant stylistic improvements.

IBM

Thomas J. Watson Research Center

Yorktown Heights, New York 10598

1. Trygve Nagell, Introduction to Number Theory, Almqvist \& Wiksell, Stockholm; Wiley, New York, 1951, Chapter V. MR 13, 207.

2. P. J. MCCARTHY, "Odd perfect numbers," Scripta Math., v. 23, 1958, pp. 43-47. MR 21 \#21.

3. H.-J. KanOLd, "Über mehrfache vollkommene Zahlen. II," J. Reine Angew. Math., v. 197, 1957, pp. 82-96. MR 18, 873.

4. K. K. NORTON, "Remarks on the number of factors of an odd perfect number," Acta Arith., v. 6, 1961, pp. 365-374. MR 26 \#4950.

5. J. B. Muskat, "On divisors of odd perfect numbers," Math. Comp., v. 20, 1966, pp. 141-144. MR 32 \#076.

6. BRYANT TUCKERMan, Odd Perfect Numbers: A Search Procedure, and a New Lower Bound of 1036, IBM Research Report RC-1925, October 20, 1967, IBM Thomas J. Watson Research Center, Yorktown Heights, N.Y. 10598. Copy deposited in UMT file.

7. BRYANT TUCKERMAN, "Odd perfect numbers: A search procedure, and a new lower bound of $10^{36}$," Notices Amer. Math. Soc., v. 15, 1968, p. 226. Abstract \#68T-116.

8. Bryant TUCKERMAN, Odd-Perfect-Number Tree to $10^{36}$, to accompany 'A Search Procedure and Lower Bound for Odd Perfect Numbers.' Copy deposited in UMT file.

9. Peter Hagis, JR., "A lower bound for the set of odd perfect numbers," Math. Comp., v. 27, 1973, pp. 951-953. 